УДК 51-74,374.1

DOI 10.52575/2687-0932-2021-48-2-376-382

\title{
Применение STEM-технологий при разработке интерактивных web-приложений
}

\author{
Синюгина О.О., Беляева И.Н., Величко М.А. \\ Белгородский государственный национальный исследовательский университет, \\ Россия, 308015, г. Белгород, ул. Победы, 85 \\ E-mail: ibelyaeva@bsu.edu.ru, velichko@bsu.edu.ru
}

\begin{abstract}
Аннотация. В статье рассматривается применение STEM-технологий в проектной деятельности на примере разработки интерактивных web-приложений, которые представляют собой учебные проекты, реализуемые школьниками на базе НИУ «БелГУ». Применение STEM-технологий именно к данному типу проектов составляет новизну исследования. Рассматривается структура и процесс создания комплексов интерактивных программ с применением математического моделирования. Описываются результаты, достигаемые с применением STEM-технологий. Уделяется внимание вопросу влияния STEM-технологий на процесс изучения HTML, CSS и JavaScript, которые являются средством разработки интерактивных web-приложений.
\end{abstract}

Ключевые слова: математическое моделирование, численные методы, комплексы программ, STEMтехнологии, проектно-исследовательская деятельность, web-технологии.

Для цитирования: Синюгина О.О., Беляева И.Н., Величко М.А. 2021. Применение STEM-технологий при разработке интерактивных web-приложений. Экономика. Информатика, 48 (2): 376-382. DOI 10.52575/ 2687-0932-2021-48-2-376-382.

\section{Implementation of STEM-technologies in the development of interactive web-applications}

\author{
Oksana O. Sinugina, Irina N. Belyaeva, Maksim A. Velichko \\ Belgorod National Research University, 85 Pobeda St, Belgorod, 308015, Russia \\ E-mail: ibelyaeva@bsu.edu.ru,velichko@bsu.edu.ru
}

\begin{abstract}
The article discusses the use of STEM-technologies in project activities by the example of the of interactive web-applications development. These web-applications are educational projects implemented by schoolchildren on the basis of Belgorod National Research University. STEM technologies are educational technologies that combine science, technology, engineering, and mathematics. The application of the abovementioned technologies to this type of projects is the novelty of the study. In these projects, web-technologies are used not only for creation of the interactive web-applications themselves, but also for the various constructions and models. The structure and process of creating complexes of interactive programs with the use of mathematical modeling are considered. The results achieved with the use of STEM technologies are described. Attention is paid to the impact of STEM technologies on the process of learning HTML, CSS and JavaScript, which are a means of interactive web-applications developing.
\end{abstract}

Keywords: mathematical modeling, numerical methods, software packages, STEM-technologies, design and research activities, web-technologies.

For citation: Sinugina O.O., Belyaeva I.N., Velichko M.A. 2021. Implementation of STEM-technologies in the development of interactive web-applications. Economics. Information technologies, 48 (2): 376-382. (in Russian). DOI 10.52575/2687-0932-2021-48-2-376-382. 


\section{Введение}

На сегодняшний день одним из требований к результатам освоения основной образовательной программы является выполнение индивидуального учебного проекта. В соответствии с ФГОС СОО «индивидуальный проект представляет собой особую форму организации деятельности обучающихся (учебное исследование или учебный проект), который выполняется обучающимся самостоятельно под руководством учителя (тьютора) по выбранной теме в рамках одного или нескольких изучаемых учебных предметов, курсов в любой избранной области деятельности» [ФГОС СОО, 2021, с. 24].

В соответствии с этим требованием в НИУ «БелГУ» был создан образовательный центр «Пролог». Данный образовательный центр направлен на реализацию школьниками своих научноисследовательских проектов. Одной из дополнительных общеобразовательных программ данного центра является курс «Проектная лаборатория с использованием STEM-технологий», который реализуется на базе факультета математики и естественно-научного образования. Обучение занимает 5 месяцев и проходит в срок с 1 ноября 2020 года по 30 марта 2021 года [Проект под..., 2020].

Перед данным курсом стоят задачи, выраженные требованиями, которые ФГОС СОО предъявляет к проектам:

«Результаты выполнения индивидуального проекта должны отражать:

сформированность навыков коммуникативной, учебно-исследовательской деятельности, критического мышления;

способность к инновационной, аналитической, творческой, интеллектуальной деятельности;

сформированность навыков проектной деятельности, а также самостоятельного применения приобретенных знаний и способов действий при решении различных задач, используя знания одного или нескольких учебных предметов или предметных областей;

способность постановки цели и формулирования гипотезы исследования, планирования работы, отбора и интерпретации необходимой информации, структурирования аргументации результатов исследования на основе собранных данных, презентации результатов» [ФГОС СОО, 2021, с. 24].

Для достижения поставленных задач были выбраны STEM-технологии ввиду того, что они не только позволяют реализовать комплекс поставленных задач, но и подходят для создания проектов в сфере естественных наук и математики.

Аббревиатура STEM расшифровывается как Science (Наука), Technology (Технологии), Engineering (инженерия) и Mathematics (математика). Существуют также и вариации этой аббревиатуры, в которых добавляется тот или иной компонент и соответствующая ему буква. Например, в аббревиатуру STEAM добавлен компонент Art, искусство, а в аббревиатуру STREM - Robotics, робототехника [Chernyavskikh et al., 2018; Kostina et al., 2019].

Основной задачей данной образовательной технологии является интеграция знаний в области естественных наук, математики, технологии и инженерии, для развития креативного и научного мышления, навыков работы в команде, а также способствованию реализации современных интересных и конкурентоспособных проектов [Velichko et al., 2018; Migal et al., 2019; Belyaeva et al., 2020; Gafurova et al., 2020].

Применение STEM-технологий помогает сформировать целостный взгляд на разрозненный изучаемый материал. Так, Лазарева С.А. и Марчук Т.Л. [2019, с. 76] подчеркивают, что «при использовании STEM-технологии мы имеем возможность осуществлять комплексный междисциплинарный подход с проектным обучением, сочетающим в себе естественные науки с технологиями, инженерией и математикой. Как и в жизни, все предметы интегрированы и взаимосвязаны в единое целое, что позволяет сформировать у учащихся целостное мировосприятие». 


\section{Результаты и их обсуждение}

В данной статье мы рассмотрим то, как можно применить данные STEM-технологии при создании интерактивных web-приложений - индивидуальных проектов школьников, выполняемых в ходе занятий курса «Проектная лаборатория с использованием STEMтехнологий». Для их реализации используется набор web-технологий HTML (HyperText Markup Language - «язык гипертекстовой разметки»), CSS (Cascading Style Sheets «каскадные таблицы стилей») и JavaScript (язык программирования) [Гольчевский, 2020].

Интерактивное приложение представляет собой комплекс небольших программ и имеет следующую структуру:

1. Титульная страница, которая содержит информацию о названии проекта и имя автора проекта. С этой страницы переход осуществляется на следующую, главную страницу.

2. Главная страница, содержащая компьютерную интерактивную модель по выбранной учеником теме. Интерактивная модель представляет собой результат математического моделирования процессов или объектов реального мира. Далее, посредством взаимодействия с содержимым этой страницы, переход можно осуществить как на теоретические страницы, так и на тестовые.

2.1. Теоретические страницы содержат в себе дополнительный теоретический материал по выбранному компоненту модели, информацию о котором пользователь решил узнать, взаимодействуя с интерактивной моделью на главной странице. Такие страницы будут созданы для каждого компонента модели, их количество определяется индивидуально.

2.2. Тестовые страницы представляют собой интерактивный тест, который служит для самопроверки пользователя после изучения главной и теоретических страниц. Данный интерактивный тест будет автоматически обрабатывать ответы и в конце прохождения показывать результат.

Рассмотрим, этапы создания такого интерактивного web-приложения и вклад STEMтехнологий при их разработке.

Первоначально требуется выбрать тему, включающую в себя разные предметные области: биология, химия, физика, астрономия, и другие или их комбинация. Следующим шагом необходимо детально изучить теоретическую информацию для самостоятельной разработки интерактивной компьютерной модели. На данном этапе возникает необходимость применения различных математических расчетов, которые нужны для реализации компьютерной модели и зависят от выбранной темы. Затем идет создание страниц с уже изученной, проанализированной и систематизированной теоретической информацией. Конечным шагом следует создание вопросов для самопроверки, которые потом станут впоследствии интерактивным тестом. Из этого следует, что данное интерактивное webприложение может использоваться не только для презентации результатов исследования, но и для самостоятельного изучения другими пользователями, а также для самопроверки любыми пользователями приложения. Помимо самостоятельной разработки данного приложения, при поддержке преподавателей и студентов-кураторов, параллельно происходит изучение и webтехнологий HTML, CSS и JavaScript, необходимых для реализации проекта.

Если мы рассмотрим содержание деятельности в процессе разработки приложения с точки зрения STEM-технологий, то можно выделить содержание каждого элемента STEMтехнологий [Kosheleva, Kreinovich, 2019; Larchenkova et al., 2019; Гребенюк, Булан, 2020; Aniskin et al., 2020]. К науке будет относиться конкретная предметная область, в рамках которой разрабатывается приложение. К технологиям следует отнести web-технологии создания web-pecyрсов, а именно HTML, CSS и JavaScript, комплексы программ для 2d- и 3dпроектирования и математического моделирования. К инженерии можно отнести процесс разработки компьютерной интерактивной модели. Для численного решения уравнений движения при построении математических моделей различных процессов и явлений использовались модификации метода Ньютона. В качестве инструментов для проведения 
численного моделирования применялся программный комплекс Mathcad (по интерфейсу и функциям он наиболее подходил для обучающихся). К математике относятся вычисления и расчеты, необходимые для создания этой модели.

Задействуется и творческий потенциал ученика, который выражается в разработке дизайна web-pecypca. Следовательно, в этом случае даже можно использовать термин STEAM-технологии [Фаенко, 2019; Власенко, Дубицкая, 2020; Колесникова, Куденко, 2020], где к основному термину добавляется Art (искусство). Следует отметить, что ученики работают с графическими редакторами для того, чтобы получить наиболее подходящие изображения для оформления своего приложения.

В процессе подготовки учащиеся углубляют свои знания в сфере естественнонаучных дисциплин, анализируя, систематизируя и организуя информацию, расширяют границы изучаемого, выходя за границы школьной программы.

В результате, обучающиеся приобретают навыки проектно-исследовательской деятельности, навыки создания моделей различных процессов и объектов, а также овладевают навыками работы с информационно-коммуникационными технологиями. Применение STEMтехнологий способствует также формированию научного мышления, креативности, формированию навыков в поиске путей и способов решения поставленных задач [Клещева, Бернавская, 2019; Лозицкий, 2019; Смирнова, Сарамуд, 2019; Елемешина, 2020].

В ходе занятий школьники взаимодействуют и со сверстниками, и со взрослыми, преподавателями университета и студентами-кураторами, что также способствует формированию коммуникативного навыка.

Нельзя не отметить и то, как проектная деятельность с использованием STEMтехнологий положительно влияет на процесс изучения web-технологий.

Во-первых, повышается мотивация в изучении этих web-технологий. В этом случае изучение HTML, CSS и JavaScript становится не самоцелью, а средством реализации проекта, что дополнительно мотивирует учащихся к овладению знаний по данной теме. Учащиеся видят, как можно реализовать свои идеи на практике, и это вносит дополнительный вклад.

Во-вторых, повышается степень закрепления изученного материала и непроизвольного запоминания. Можно сравнить изучение HTML, CSS и JavaScript в ходе выполнения разрозненных заданий, не объединенных общей темой, и изучение HTML, CSS и JavaScript в ходе разработки единого проекта, решения задач которого объединены общей тематикой и связаны между собой. В первом случае, хоть и решаются поставленные задачи и происходит изучение и закрепление нового материала, однако велика вероятность того, что с течением времени изученный материал забудется, так как он может больше и не использоваться. Кроме этого, рассматривая данный случай, ученик может получить неактуальную на данном этапе для него информацию, которая может редко использоваться самим учеником, так как для него нет в этом необходимости. Напротив, если мы рассмотрим второй вариант, при котором изучение теории HTML, CSS и JavaScript проходит с параллельным созданием целостного проекта, то в этом случае информация будет усваиваться лучше, так как изученный материал актуален для ученика и постоянно используется. При создании проекта ученику каждый раз придется обращаться уже к созданному варианту проекта и дополнять его, и при этом ученик будет вспоминать и то, что он уже сделал. И в результате изученный материал будет постепенно закрепляться в ходе многочисленных повторений.

\section{Заключение}

Таким образом, применение STEM-технологий не только помогает решить поставленные ФГОС СОО требования перед выполнением индивидуального учебного проекта, но также развивает ряд компетенций обучающихся, которые заложены в образ современного школьника. К таким компетенциям относится овладение навыками обработки информации, навыками проектной и исследовательской деятельности, навыками работы с ИКТ технологиями, коммуникативными навыками. Применение этих технологий расширяет кругозор школьника и 
углубляет знания из различных областей, а также помогает в реализации школьниками своих творческих способностей и в формировании целостного мировоззрения, которое позволит в будущем расширить диапазон задач, которые смогут решать ученики.

\section{Список литературы}

1. Власенко А.П., Дубицкая Л.В. 2020. Синтез SCRUM и STEM технологий при изучении физики в школе. Физика в школе, 1: 34-37.

2. Гольчевский Ю.В. 2020. Подходы к проектированию и разработке современного корпоративного web-pесурса. Экономика. Информатика, 47 (2): 432-440.

3. Гребенюк Т.Б., Булан И.Г. 2020. Использование STEM-подхода в условиях среднего профессионального образования. Известия Балтийской государственной академии рыбопромыслового флота: психолого-педагогические науки, 1 (51): 22-29.

4. Елемешина Н.Н. 2020. Развитие STЕМ-лаборатории в дошкольной образовательной организации. В сборнике: Горизонты и риски развития образования в условиях системных изменений и цифровизации. Сборник научных трудов XII Международной научно-практической конференции в 2 частях: 188-192.

5. Клещева Н.А., Бернавская М.В. 2019. Метапредметный подход к разработке учебных курсов для STEM-специальностей. Общество: социология, психология, педагогика, 12 (68): 131-135.

6. Колесникова Е.M., Куденко И.А. 2020. Интерес к STEM-профессиям в школе: проблемы профориентации. Социологические исследования, 4: 124-133.

7. Лазарева С.А., Марчук Т.Л. 2019. SТЕМ-технология как средство формирования инженерного мышления школьников. Пермский педагогический журнал, 10: 76-79.

8. Лозицкий В.Л. 2019. Педагогический потенциал средств STЕМ-образования. Выхаванне i дадатковая адукацыя, 6: 21-25.

9. Проект под руководством ученых БелГУ. 2020. URL: https://shuhov-lycee.ru/life/news/proektpod-rukovodstvom-uchenykh-belgu/ (дата обращения: 14.02.2021).

10. Смирнова Н.А., Сарамуд И.А. 2019. Математика как база STЕМ-образования школьника. В сборнике: Сетевое образовательное взаимодействие в подготовке педагога информационного общества. Международная научно-практическая конференция: 435-441.

11. Фаенко А.В. 2019. К вопросу об актуальности STЕМ-образования. В сборнике: Физикоматематическое образование: цели, достижения и перспективы. Материалы Международной научнопрактической конференции. Белорусский государственный педагогический университет имени Максима Танка: 189-191.

12. Федеральный государственный образовательный стандарт среднего общего образования. 2016-2021. URL: https://fgos.ru/ (дата обращения: 14.02.2021).

13. Aniskin V., Korostelev A., Busygina A., Kurochkin A., Sobakina T. 2020. Teaching potential of integrated learning technologies SMART, STEM and STEAM. Revista de la Universidad del Zulia, 11 (29): 328-336.

14. Belyaeva I., Chekanov N., Chekanova N., Kirichenko I., Ptashny O., Yarkho T. 2020. Calculation of the Green's function of boundary value problems for linear ordinary differential equations. EasternEuropean Journal of Enterprise Technologies, 1 (4 (103)): 43-52.

15. Chernyavskikh S.D., Velichko M.A., Kostina I.B., Krasovskaya L.V., Satler O.N. 2018. Improving educational process quality in the lessons of natural and mathematical cycle by means of stem-training. Cypriot Journal of Educational Sciences, 13 (4): 501-510.

16. Gafurova N.V., Osipova S.I., Shubkina O. Yu., Kublitskaya Yu. G., Arnautova E.A. 2020. Collective subject in implementation of STEM technologies in engineering education. Theory and Practice of Project Management in Education: Horizons and Risks, 79: 2006.

17. Kosheleva O., Kreinovich V. 2019. Why STEM? Mathematical Structures and Modeling, 2 (50): 99-106.

18. Kostina I.B., Gladkikh Y.P., Velichko M.A., Krasovskaya L.V., Satler O.N. 2019. Assessment of ecological consciousness formation among adolescent girls in the learning process by means of specialized computer systems. International Journal of Recent Technology and Engineering, 8 (2 Special Issue 10): 665-668.

19. Larchenkova L., Gavronskaya Y., Rogovaya O. 2019. Critical thinking in STEM (science, technology, engineering, and mathematics). Utopia y Praxis Latinoamericana, 24 (Extra 6): 32-41. 
20. Migal L.V., Bondarev V.G., Bondareva T.P., Belyaeva I.N. 2019. Mathematical model of coordination number of spherical packing. Compusoft, 8 (6): 3187-3191.

21. Velichko M.A., Satler O.N., Krasovskaya L.V., Kostina I.B., Gladkikh, Y.P. 2018. Calibration of ultrasonic flowmeter on Wi-Fi network using a web browser. Journal of Advanced Research in Dynamical and Control System, 10 (8): 1593-1596.

\section{References}

1. Vlasenko A.P., Dubickaya L.V. 2020. Synthesis of SCRUM and STEM technologies when studying physics at school. Fizika v shkole, 1: 34-37.

2. Gol'chevskij Ju.V. 2020. Approaches to the modern corporate web resource design and development. Economics. Information technologies, 47 (2): 432-440 (in Russian).

3. Grebenyuk T.B., Bulan I.G. 2020. Use of the STEM-approach in the conditions of secondary vocational education. Izvestiya Baltijskoj gosudarstvennoj akademii rybopromyslovogo flota: psihologopedagogicheskie nauki, 1 (51): 22-29.

4. Elemeshina N.N. 2020. 'Razvitie STEM- laboratorii v doshkol'noj obrazovatel'noj organizacii [Development of a STEM laboratory in a preschool educational organization.]. In: Gorizonty i riski razvitiya obrazovaniya v usloviyah sistemnyh izmenenij i cifrovizacii. Sbornik nauchnyh trudov XII Mezhdunarodnoj nauchno-prakticheskoj konferencii v 2-uh chastyah: 188-192.

5. Kleshcheva N.A., Bernavskaya M.V. 2019. Meta-subject approach to the development of training courses for STEM specialties. Obshchestvo: sociologiya, psihologiya, pedagogika, 12 (68): 131-135.

6. Kolesnikova E.M., Kudenko I.A. 2020. Interest for STEM professions at school: problems of career guidance. Sociologicheskie issledovaniya, 4: 124-133.

7. Lazareva C. A., Marchuk T. L. 2019. Stem-tehnologija kak sredstvo formirovanija inzhenernogo myshlenija shkol'nikov [STEM technology as a means of shaping engineering thinking in schoolchildren]. Permskij pedagogicheskij zhurnal, 10: 76-79.

8. Lozickij V.L. 2019. Pedagogicheskij potencial stedstv STEM-obrazovaniya [Pedagogical potential of STEM education]. Vyhavanne i dadatkovaya adukacyya, 6: 21-25.

9. Proekt pod rukovodstvom uchenyh BelGU [Project led by scientists from BelSU]. 2020. Available at: https://shuhov-lycee.ru/life/news/proekt-pod-rukovodstvom-uchenykh-belgu/ (accessed: 14.02.2021).

10. Smirnova N.A., Saramud I.A. 2019. Matematika kak baza STEM-obrazovaniya shkol'nika [Mathematics as the basis of STEM education for schoolchildren]. In: Setevoe obrazovatel'noe vzaimodejstvie $\mathrm{v}$ podgotovke pedagoga informacionnogo obshchestva. Mezhdunarodnaya nauchno-prakticheskaya konferenciya: 435-441.

11. Faenko A.V. 2019. K voprosu ob aktual'nosti STEM-obrazovaniya [To the question of the relevance of STEM education.]. In: Fiziko-matematicheskoe obrazovanie: celi, dostizheniya i perspektivy. Materialy Mezhdunarodnoj nauchno-prakticheskoj konferencii. Belorusskij gosudarstvennyj pedagogicheskij universitet imeni Maksima Tanka: 189-191.

12. Federal'nyj gosudarstvennyj obrazovatel'nyj standart srednego obshhego obrazovanija [Federal State Educational Standard of Secondary General Education]. 2016-2021. Available at: https://fgos.ru/ (accessed: 14.02.2021).

13. Aniskin V., Korostelev A, Busygina A, Kurochkin A, Sobakina T. 2020. Teaching potential of integrated learning technologies SMART, STEM and STEAM. Revista de la Universidad del Zulia, 11 (29): 328-336.

14. Belyaeva I., Chekanov N., Chekanova N., Kirichenko I., Ptashny O., Yarkho T. 2020. Calculation of the Green's function of boundary value problems for linear ordinary differential equations. EasternEuropean Journal of Enterprise Technologies, 1 (4 (103)): 43-52.

15. Chernyavskikh S.D., Velichko M.A., Kostina I.B., Krasovskaya L.V., Satler O.N. 2018. Improving educational process quality in the lessons of natural and mathematical cycle by means of stem-training. Cypriot Journal of Educational Sciences, 13(4): 501-510.

16. Gafurova N.V., Osipova S.I., Shubkina O. Yu., Kublitskaya Yu. G., Arnautova E.A. 2020. Collective subject in implementation of STEM technologies in engineering education. Theory and Practice of Project Management in Education: Horizons and Risks, 79: 2006.

17. Kosheleva O., Kreinovich V. 2019. Why STEM? Mathematical Structures and Modeling, 2 (50): 99-106. 
18. Kostina I.B., Gladkikh Y.P., Velichko M.A., Krasovskaya L.V., Satler O.N. 2019. Assessment of ecological consciousness formation among adolescent girls in the learning process by means of specialized computer systems. International Journal of Recent Technology and Engineering, 8 (2 Special Issue 10): 665-668.

19. Larchenkova L., Gavronskaya Y., Rogovaya O. 2019. Critical thinking in STEM (science, technology, engineering, and mathematics). Utopia y Praxis Latinoamericana, 24 (Extra 6): 32-41.

20. Migal L.V., Bondarev V.G., Bondareva T.P., Belyaeva I.N. 2019. Mathematical model of coordination number of spherical packing. Compusoft, 8 (6): 3187-3191.

21. Velichko M.A., Satler O.N., Krasovskaya L.V., Kostina I.B., Gladkikh, Y.P. 2018. Calibration of ultrasonic flowmeter on Wi-Fi network using a web browser. Journal of Advanced Research in Dynamical and Control System, 10 (8): 1593-1596.

\section{ИНФОРМАЦИЯ ОБ АВТОРАХ}

Беляева Ирина Николаевна, кандидат физикоматематических наук, доцент кафедры информатики, естественно-научных дисциплин и методик преподавания Белгородского государственного национального исследовательского университета, Белгород, Россия

Величко Максим Андреевич, кандидат физикоматематических наук, доцент кафедры информатики, естественно-научных дисциплин и методик преподавания Белгородского государственного национального исследовательского университета, Белгород, Россия

Синюгина Оксана Олеговна, студентка 5 курса, по направлению подготовки 44.03.05 Педагогическое образование, профиль Информатика и иностранный язык (английский), Белгородский государственный национальный исследовательский университет, г. Белгород, Россия

\section{INFORMATION ABOUT THE AUTHORS}

Irina N. Belyaeva, Candidate of Physical and Mathematical Sciences, Associate Professor (Docent) of the Department of Computer Science, Natural Sciences and Teaching Methods Belgorod National Research University, Belgorod, Russia

Maksim A. Velichko, Candidate of Physical and Mathematical Sciences, Associate Professor (Docent) of the Department of Computer Science, Natural Sciences and Teaching Methods Belgorod National Research University, Belgorod, Russia

Oksana O. Sinugina, 5th year student of 44.03 .05 curricula Pedagogical Education Computer Science and foreign Language (English), Belgorod National Research University, Belgorod, Russia 